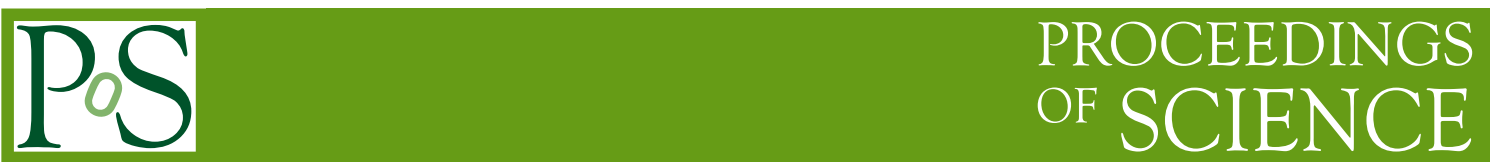

\title{
RFI measurements at the WSRT
}

\section{Hans van der Marel*}

Radio Observatory Division, ASTRON, Oude Hoogeveensedijk 4, 7991 PD Dwingeloo, The Netherlands

E-mail: mareleastron.nl

\section{Pieter Donker}

Radio Observatory Division, ASTRON, Oude Hoogeveensedijk 4, 7991 PD Dwingeloo, The Netherlands

E-mail: donker@astron.nl

In order to minimize the effects of RFI and RFI mitigation systems on astronomical observations it is mandatory to have as little RFI at an observatory as possible. At the WSRT we try to establish this by continuously monitoring the RFI from outside the observatory with a dedicated spectrum monitoring station and minimizing the amount of RFI produced by equipment at the observatory. In this paper emphasis will be put in the spectrum monitoring functionality as part of the RFI measurements.

RFI mitigation workshop

29-31 March 2010

Groningen, the Netherlands

\footnotetext{
* Speaker.
} 


\section{Introduction}

When doing radio astronomical observations outside the allocated bands it is important to know what kind of interference can be expected. Especially for frequencies outside the bands that are allocated to radio astronomy that are not observed very frequently, it is very helpful to know which frequencies are not used by other users, or are used infrequently. This can be important for the preparation of observations, but also for the analysis of the observations. Spectrum monitoring is an important tool to estimate the amount of global interference.

At the WSRT spectrum monitoring is done on a regular base for many years. To extend the frequency range and to improve reliability of the spectrum monitoring station at the WSRT a new system has been realised in 2005. A modification in the antenna set-up was done in the end of 2007. Since the start of its operation the station has gathered an enormous amount of valuable data. This paper describes the spectrum monitoring station in its current configuration.

\section{The spectrum monitoring system}

The spectrum monitoring station at the WSRT is continuously scanning the spectrum. The hardware consists of a number of commercially available items. The control and analysis software has been developed specifically for this application, but has been proven useful for other applications as well.

\subsection{Set-up of Monitoring Station}

In Figure 1 a schematic overview of the spectrum monitoring system is shown.

The key hardware components of the monitoring station are:
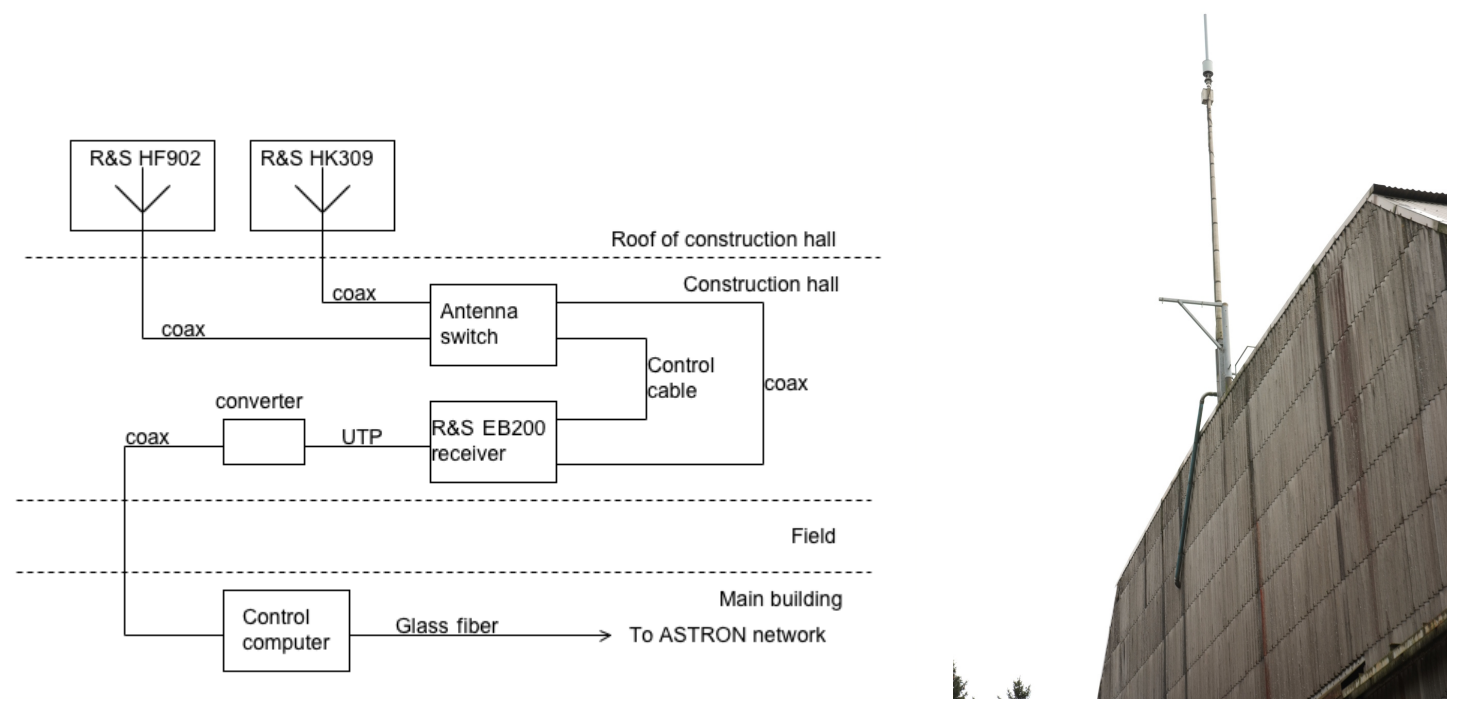

Figure 1: Schematic overview of the spectrum monitoring station of the WSRT (left) and a photograph of the antenna tower on the roof of the construction hall (right). 
- Rohde \& Schwarz EB 200 miniport receiver [1]

- Frequency range: $10 \mathrm{kHz}-3 \mathrm{GHz}$

- Noise floor: $-117 \mathrm{dBm}$ at $30 \mathrm{kHz}$ bandwidth

- Scan speed: 126 points/s $\Longrightarrow 20-3000 \mathrm{MHz}$ in 13.5 minutes at $30 \mathrm{kHz}$

- Rohde \& Schwarz HK309 omnidirectional passive antenna [2]

- Frequency range: 20 - $1300 \mathrm{MHz}$

- linear polarization

- Rohde \& Schwarz HF902 omnidirectional passive antenna [3]

- Frequency range: 1 - $3 \mathrm{GHz}$

- Dual linear polarizations ( $\mathrm{V}$ is being used)

The antennas of the monitoring station are placed in a tower on the roof of the construction hall (see Figure 1) between telescopes 1 and 2 of the Westerbork array. The total height of the tower is approximately 35 meters above the ground, which means that the antennas have a free view over the tops of the forest that surrounds the WSRT. The Rohde \& Schwarz EB200 receiver is placed in a shielded box just below the roof of the construction hall. It is controlling a coax switch with which the right antenna for the measured frequency can be selected. The receiver in turn is controlled by a computer in the main building of the WSRT over a coaxial Ethernet cable. Because the receiver only has a UTP Ethernet connection a converter for UTP to coax is placed in the same shielded box to bridge the 800 meters to the main building. The control computer is connected to the ASTRON network through glass fiber.

\subsection{Control and Measurement Software}

A schematic of the control program is shown in Figure 2. Through a graphical user interface the station module and the measurement module are controlled.

The station module interacts with the station.cfg file, which contains the information about the configuration of the monitoring station such as the location of the station, information about the receiver and antennas that are used and which calibration files have to be used. It also uses the calibration files, containing the calibration data for the antennas, the receivers, and if applicable, for the filters and amplifiers that are used.

The measurement module gets information about the station configuration from the station module and information about settings for this particular measurement from the measure.dat file. The measurement module also controls the EB200 receiver through the EB200 module and receives the measured data through the same module from the receiver. The measurement module also passes information about the status of the measurement to the GUI.

All measured data as well as configuration and calibration data is send to the RMDF module. This module writes the data in three separate files according to the Radio Frequency Interference Monitoring Data Format (RMDF). The *.rmh file is a header file, containing configuration data as well as time stamps for the scans. The *.rmk file contains the calibration data for the used set-up 


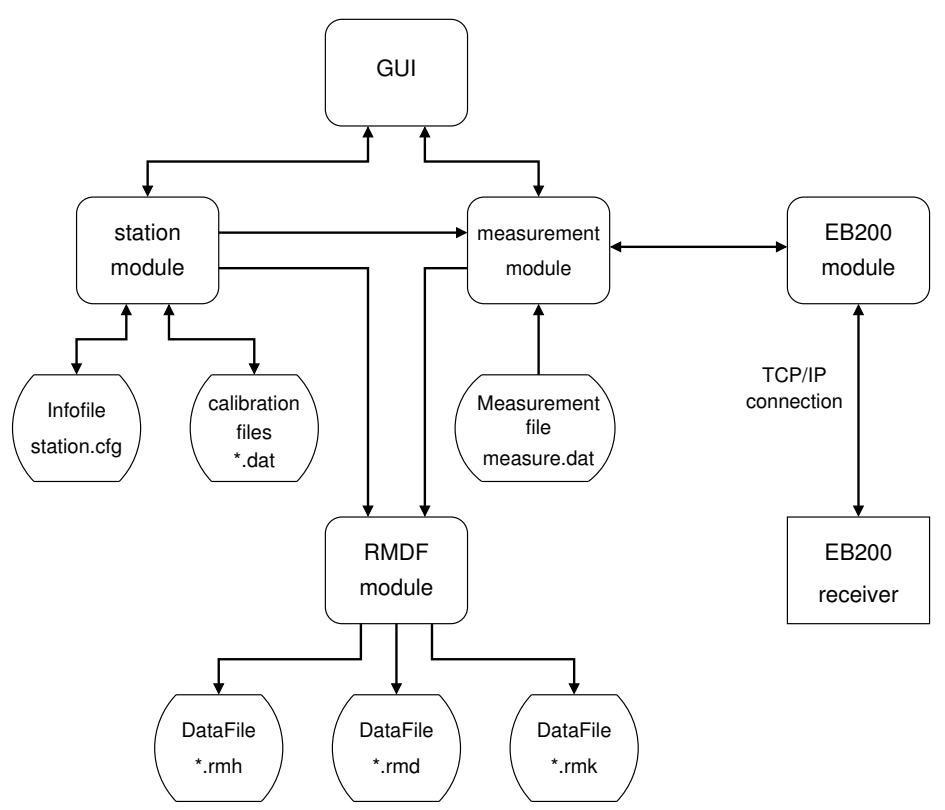

Figure 2: Schematic of the control program

and settings and the *.rmd file contains the measured data points. For every measurement these three files are written to disk. Typically one measurement is performed every 24 hours.

\subsection{Analysis Software}

The analysis of the spectrum monitoring data is performed off-line with help of the SpectrumViewer program. The schematic of this program is shown in Figure 3. The Spectrum unit of the analysis program retrieves the data files over the network from the central repository or from the local disk. The measured data is calibrated using the information in the calibration file and formatted using the information in the header file. Then everything is sent to the Graph unit which presents the spectrum data in graphical form on the screen. It is also possible to save the spectral data in a bitmapped file.

The GUI of the SpectrumViewer program is shown in Figure 4. From top to bottom the fol-

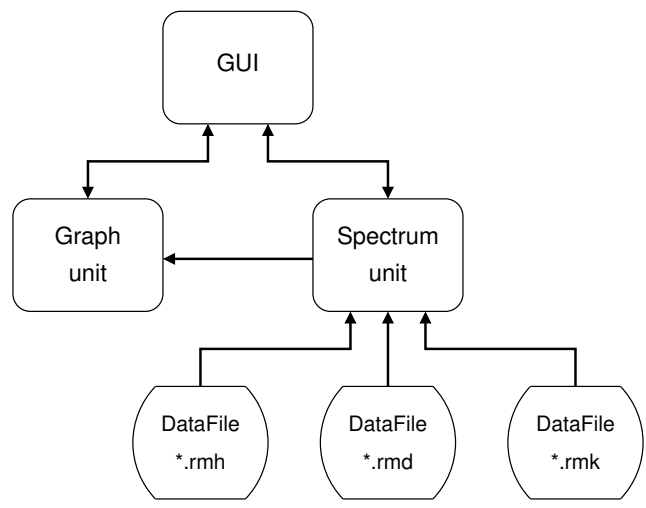

Figure 3: Schematic of the analysis program. 


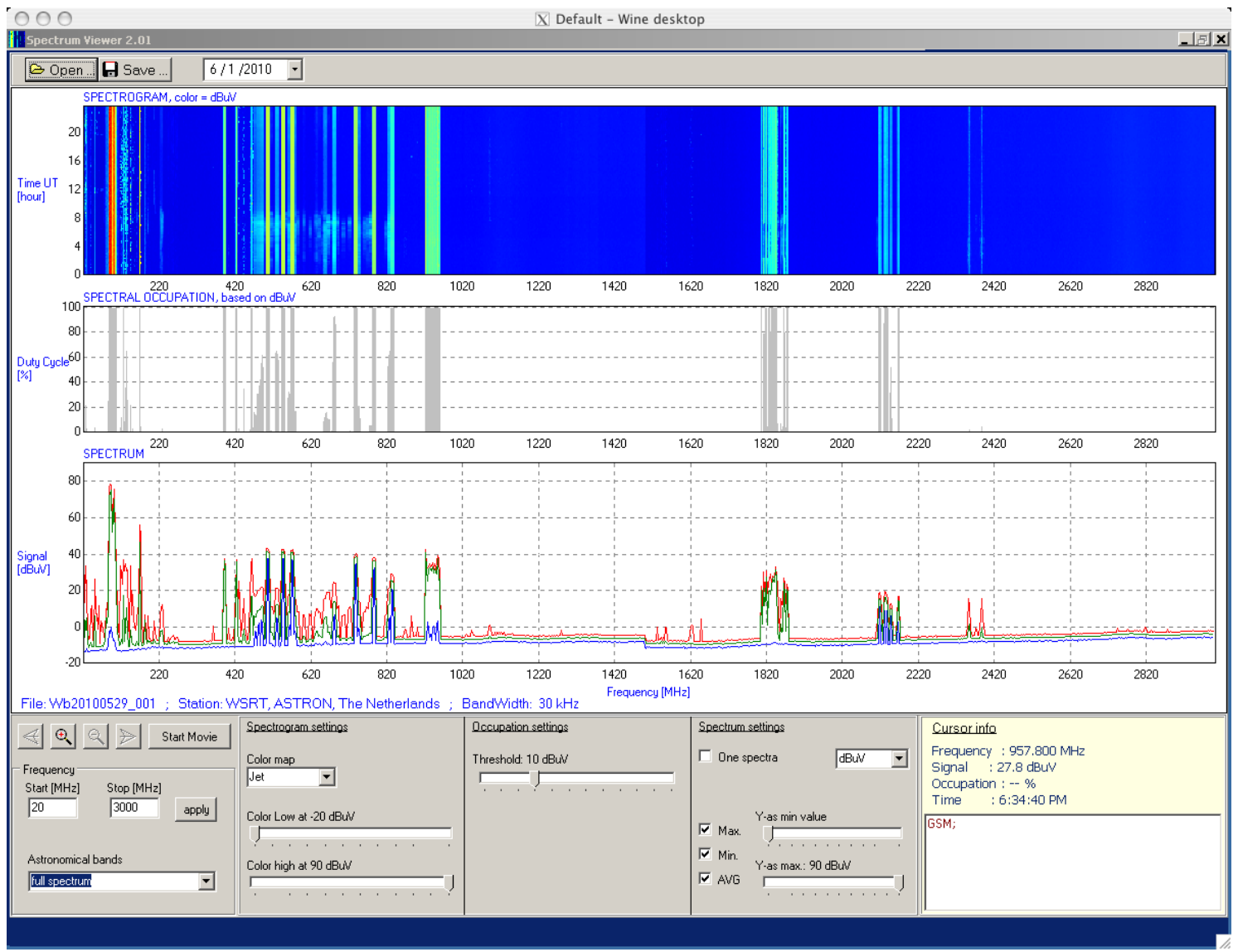

Figure 4: Graphical User Interface of the SpectrumViewer program.

lowing spectral data is shown in the GUI: a spectrogram, or waterfall plot, the spectral occupation above a certain threshold and a spectrum with the minimum, maximum and average value of the signal strength.

Completely at the top of the GUI one can select the monitoring data that one wants to analyse. At the bottom of the GUI several settings can be made for the plots, such as the frequency range that should be displayed, color settings for the spectrogram, the threshold for the occupation plot and scaling for the spectrum plot and spectrogram. Also the unit for the signal strength (in $\mathrm{dBm}$, $\mathrm{dB} \mu \mathrm{V}$ or $\mathrm{dB} \mu \mathrm{V} / \mathrm{m}$ ) can be selected. A small window in the lower right corner shows information about the data at the cursor position.

The analysis software also provides a spectrum of the last sweep that has been made and which can be viewed on an internal web page of ASTRON.

\section{Conclusions}

The spectrum monitoring station at the WSRT provides lots of valuable data for the operators, technicians and astronomers that use the WSRT. The data can be used to predict whether it is feasable to make a radio astronomical observation at a frequency outside the allocated bands. But it can also be used to find out about interference when analysing the astronomical observation (also 


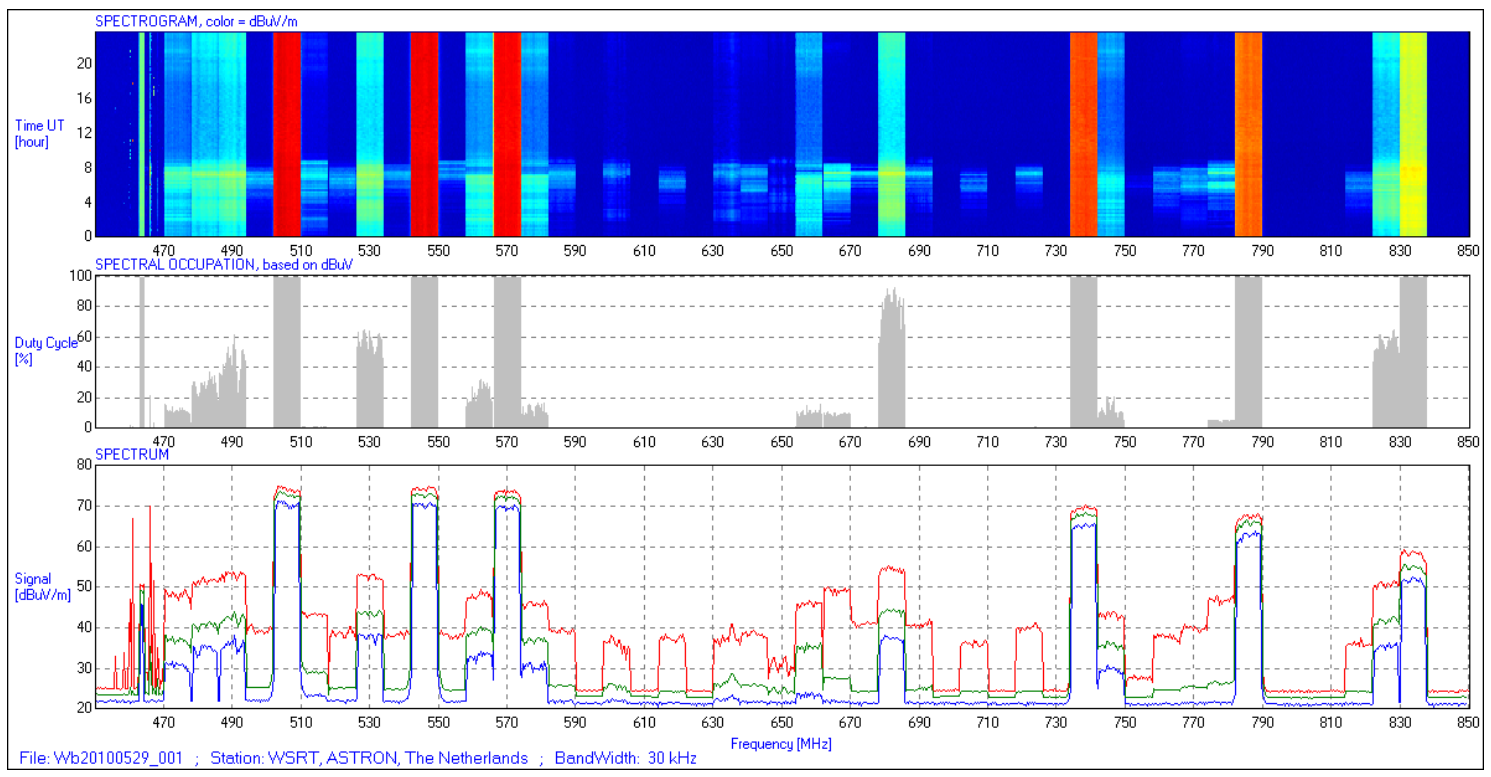

Figure 5: Monitoring data of the UHF band showing the many digital TV channels.

inside allocated bands). In Figure 5 there is an example of the spectrum of the UHF band. In the Netherlands TV channel $38(610 \mathrm{MHz})$ is (still) kept free for radio astronomical observations and transmitters at channel $39(618 \mathrm{MHz})$ are placed far away from the WSRT. So, usually it is possible to observe at channels 38 and 39, but this particular day atmospheric conditions advanced the transmission of the signals over much larger distances than usual at around 8 in the morning.

Since the spectrum monitoring station was installed it is gathering spectral data 24 hours per day, 7 days per week unattended. Apart from some down time due to maintenance it has proven to be very stable.

Due to the use of omnidirectional antennas it is not possible to find out from which direction signals are coming. However, the advantage is that all signals are received. The currently used Rohde \& Schwarz receiver is a scanning receiver. That means that only once every 13.5 minutes it measures at a certain frequency. The consequence is that quickly changing signals are not measured properly and can even be missed completely. So, some improvements can still be made.

\section{References}

[1] http://www2.rohde-schwarz.com/product/EB200.html

[2] http://www2.rohde-schwarz.com/product/HK309.html

[3] http://www2.rohde-schwarz.com/product/HF902.html 